\title{
Robust Exponential Stabilization of Uncertain Impulsive Bilinear Time-Delay Systems with Saturating Actuators
}

\author{
Yang Shujie, Shi Bao, Zhang Qiang, and Pan Tetie \\ Institute of System Science and Mathematics, Naval Aeronautical and Astronautical University, Yantai, Shandong 264001, China \\ Correspondence should be addressed to Yang Shujie, yangshujie@163.com \\ Received 24 September 2010; Accepted 14 November 2010 \\ Academic Editor: Onur Toker \\ Copyright () 2010 Yang Shujie et al. This is an open access article distributed under the Creative Commons Attribution License, \\ which permits unrestricted use, distribution, and reproduction in any medium, provided the original work is properly cited.
}

This paper investigates the problem of robust exponential stabilization for uncertain impulsive bilinear time-delay systems with saturating actuators. By using the Lyapunov function and Razumikhin-type techniques, two classes of impulsive systems are considered: the systems with unstable discrete-time dynamics and the ones with stable discrete-time dynamics. Sufficient conditions for robust stabilization are obtained in terms of linear matrix inequalities. Numerical examples are given to illustrate the effectiveness of the theoretical results.

\section{Introduction}

In practical control systems, impulsive dynamical systems are very important and have attracted considerable interest in science and engineering during the past decades. Two classical monographs are Lakshmikantham et al. [1] and Bainov and Simeonov [2]. In general, as reported in [1], impulsive systems provide a natural framework for mathematical modeling of many real-world evolutionary processes where the states undergo abrupt changes at certain instants or at variable instants, or autonomous systems with impulsive effects. Stability properties of impulsive systems have been extensively studied in the literatures. We refer to Bainov and Simeonov [2], Li, Soh and $\mathrm{Xu}[3,4], \mathrm{Li}$, Wen and Soh [5], Yang [6], Chen and Zheng [7], Barreiro and Baños [8], and the references therein. The impulsive control method based on stability theory of impulsive dynamical systems has been widely used, see [7], Xu and Teo [9], and Liu, Eberhard and Teo [10].

Saturating nonlinear actuators is one of the common nonlinearities and exists in many practical systems. If a control system is designed to be stable without considering the saturation, the stability of the closed-loop-controlled system cannot be guaranteed, see Ma and Zhang [11]. Two important papers on saturation control are Full [12] and Sontag and Sussmann [13]. However, as we have known, no papers have considered the saturation control in impulsive systems.

This work is inspired by $\mathrm{Wu}$ and Wei [7], in which the authors considered the problems of robust stability and stabilization for uncertain impulsive time-delay systems. Unfortunately, they need all the impulsive time sequences to satisfy some strict conditions. That is, the length of the intervals between two adjacent time instants must have upper bound or lower bound. But in practical systems, it is always impossible or difficult to obtain it. In this paper, robust stabilization for uncertain impulsive time-delay systems with saturating actuators is considered. With saturation control, new conditions based on Lapunov-Razumikhin function and LMIs are established which can easily be used for systems with any time sequences.

\section{Problem Formulation and Preliminaries}

Throughout this paper, if not explicitly given, matrices are assumed to have compatible dimensions. For symmetric matrices $A$ and $B$, the notation $A \geq B(A>B, A \leq B$, $A<B$ ) means $A-B$ is positive semidefinite (positive definite, negative semidefinite, negative definite) matrix. $\lambda_{\max }(\cdot)$ and $\lambda_{\min }(\cdot)$ represent the maximum and minimum eigenvalues of the corresponding matrix, respectively. $\|\cdot\|$ denotes 
Euclidean norm for vectors or the spectral norm of matrices. $P C\left([-\tau, 0], \mathbb{R}^{n}\right)$ denotes the set of piecewise right continuous function $\phi:[-\tau, 0] \rightarrow \mathbb{R}^{n}$ with the norm defined by $\|\phi\|_{\tau}=$ $\sup _{-\tau \leq \theta \leq 0}\|\phi(\theta)\|$, where $\tau$ is a known positive constant.

Consider uncertain bilinear impulsive systems with timedelay and saturating actuators

$$
\begin{gathered}
\dot{x}(t)=A(t) x(t)+A_{1}(t) x(t-\tau(t))+B(t) \operatorname{sat}(u(t)), \quad t \neq t_{k}, \\
\Delta x(t)=(C(t)-I) x\left(t^{-}\right)+\sum_{i=1}^{m} \operatorname{sat}\left(u_{i}(t)\right) B_{i}(t) x\left(t^{-}\right), \quad t=t_{k}, \\
x\left(t_{0}+\theta\right)=\phi(\theta), \quad t_{0}=0, \theta \in[-\tau, 0],
\end{gathered}
$$

where $x(t) \in \mathbb{R}^{n}$ is the system state, $u(t) \in \mathbb{R}^{m}$ is the continuous control input, $0 \leq \tau(t) \leq \tau, \Delta x\left(t_{k}\right)=x\left(t_{k}^{+}\right)-$ $x\left(t_{k}^{-}\right)$describes the state jumping at impulsive time instant $t=t_{k}$ with $x\left(t_{k}^{+}\right)=x\left(t_{k}\right)=\lim _{h \rightarrow 0+} x\left(t_{k}+h\right), x\left(t_{k}^{-}\right)=$ $\lim _{h \rightarrow 0-} x\left(t_{k}+h\right), k \in \mathbb{N}=\{1,2, \ldots\}$ and $0<t_{1}<t_{2}<\cdots<$ $t_{k}<\cdots\left(t_{k} \rightarrow \infty\right.$ as $\left.k \rightarrow \infty\right) . A(t), A_{1}(t), B(t), B_{i}(t)(i=$ $1,2, \ldots, m)$, and $C(t)$ are matrix functions with time-varying uncertainties $\Delta A(t), \Delta A_{1}(t), \Delta B(t), \Delta B_{i}(t)(i=1,2, \ldots, m)$ and $\Delta C(t)$. In this paper, we impose the following hypothesis on the above uncertainties:

$$
\begin{gathered}
\left(\Delta A(t), \Delta A_{1}(t), \Delta B(t)\right)=D_{1} F_{1}(t)\left(N_{A}, N_{A 1}, N_{B}\right), \\
\left(\Delta C(t), \Delta B_{i}(t)\right)=D_{2} F_{2}(t)\left(N_{C}, N_{B i}\right), \quad(i=1,2, \ldots, m),
\end{gathered}
$$

where $D_{1}, D_{2}, N_{A}, N_{A 1}, N_{B}, N_{C}, N_{B i}(i=1,2, \ldots, m)$ are known real constant matrices and $F_{i}(t)$ are unknown matrix functions satisfying $\left\|F_{i}(t)\right\| \leq 1$ with the elements of $F_{i}(t)$ is lebesgue measurable, $(i=1,2)$. The saturating function $\operatorname{Sat}(u(t))$ is defined as

$$
\begin{aligned}
& \operatorname{Sat}(u(t))=\left(\operatorname{sat}\left(u_{1}(t), \ldots, \operatorname{sat}\left(u_{m}(t)\right)\right)^{T},\right. \\
& \operatorname{sat}\left(u_{j}(t)\right)= \begin{cases}-U_{j}, & u_{j}(t) \leq-U_{j}, \\
u_{j}(t), & -U_{j}<u_{j}(t)<U_{j}, \\
U_{j}, & u_{j}(t) \geq U_{j},\end{cases}
\end{aligned}
$$

where $U_{j}>0(j=1,2, \ldots, m)$ are known real constants representing the upper bound of the control.

In the following, we will divide two cases to consider the robust stabilization of system (1). We denote by $\mathcal{N}_{\min }(\beta)$ the class of impulsive time sequences that satisfy $\inf _{k}\left\{t_{k}-\right.$ $\left.t_{k-1}\right\} \geq \beta$ and denote by $\mathcal{N}_{\max }(\beta)$ the class of impulsive time sequences that satisfy $\sup _{k}\left\{t_{k}-t_{k-1}\right\} \leq \beta$.

We need the following lemmas.

Lemma 1 (see Su, Chu, Lu and Ji [14]). For any vectors $x, y \in$ $\mathbb{R}^{n}$ and real matrices $A, D, E, F$ and $P>0$ with $F^{T} F \leq I$, the following inequalities hold for any scalar $\mathcal{E}>0$ :

(1) $2 x^{T} D F E y \leq(1 / \varepsilon) x^{T} D D^{T} x+\varepsilon y^{T} E^{T} E y$,

(2) $2 x^{T} y \leq x^{T} P^{-1} x+y^{T} P y$ and for $P-\varepsilon D D^{T}>0$,

(3) $(A+D F E)^{T} P^{-1}(A+D F E) \leq(1 / \varepsilon) E^{T} E+A^{T}(P-$ $\left.\varepsilon D D^{T}\right)^{-1} A$.
Lemma 2 (Schur complement [14]). For given symmetric matrix $S=\left(\begin{array}{ll}S_{11} & S_{12} \\ S_{21} & S_{22}\end{array}\right)$ with $S_{11} \in \mathbb{R}^{r \times r}$, the following three conditions are equivalent:

(1) $S<0$,

(2) $S_{11}<0, \quad S_{22}-S_{12}^{T} S_{11}^{-1} S_{12}<0$,

(3) $S_{22}<0, \quad S_{11}-S_{12} S_{22}^{-1} S_{12}^{T}<0$.

\section{Robust Stabilization}

In this section, we consider the robust stabilization of system (1).

Definition 3. System (1) with $F_{i}(t)=0(i=1,2)$ and $u(t)=0$ is said to be exponentially stable over a given class $\mathcal{N}$ if there are scalars $\varepsilon>0, \delta>0$ and $v>0$ such that $\phi \in P C\left([-\tau, 0], \mathbb{R}^{n}\right)$ with $\|\phi\|_{\tau} \leq \delta$ implying $\left\|x\left(t ; t_{0}, \phi\right)\right\|<$ $\varepsilon \exp \left(-v\left(t-t_{0}\right)\right)$ for $t \geq t_{0}$.

Definition 4. System (1) is said to be robustly exponentially stabilizable over a given class $\mathcal{N}$ with respect to static state feedback if there exists a memoryless linear state feedback law, such that the corresponding closed-loop system is robustly exponentially stable for all uncertainties satisfying (2).

For any $\phi \in P C\left([-\tau, 0], \mathbb{R}^{n}\right)$, we denote $x\left(t ; t_{0}, \phi\right)$ by $x(t)$. Let us now consider designing a memoryless state feedback control law of the form

$$
u(t)=2 K x(t)
$$

to stabilize system (1), where $2 K$ is a constant gain to be designed.

Now we decompose the saturation nonlinear function $\operatorname{Sat}(u(t))$ as the sum of a linear part and another nonlinear part (dead zone nonlinearity) $\Delta u(t)$ as $\operatorname{Sat}(u(t))=u(t)+$ $\Delta u(t)$, then by [14], (5) implies that

$$
\Delta u(t)^{T} \Delta u(t) \leq x^{T}(t) K^{T} K x(t) .
$$

Substituting (5) into system (1), we have

$$
\begin{aligned}
& \dot{x}(t)=(A(t)+2 B(t) K) x(t)+A_{1}(t) x(t-\tau(t))+B(t) \Delta u(t), \\
& t \neq t_{k} \\
& \Delta x(t)=(C(t)-I) x\left(t^{-}\right)+\sum_{i=1}^{m} \operatorname{sat}\left(u_{i}(t)\right) B_{i}(t) x\left(t^{-}\right), \quad t=t_{k}, \\
& x\left(t_{0}+\theta\right)=\phi(\theta), \quad t_{0}=0, \quad \theta \in[-\tau, 0],
\end{aligned}
$$

Lemma 5. Assume that there exist matrix $X>0$ and positive scalars $\varepsilon_{1}, \varepsilon_{2}$, $\mu$ such that 


$$
\left(\begin{array}{ccccccc}
-\mu X & X N_{C}^{T} & X C^{T} & X & 0 & \cdots & 0 \\
* & -\varepsilon_{1} I & 0 & 0 & \sqrt{U_{1}} N_{B 1} & \cdots & \sqrt{U_{m}} N_{B m} \\
* & * & \varepsilon_{1} D_{2} D_{2}^{T}-X & 0 & \sqrt{U_{1}} B_{1} & \cdots & \sqrt{U_{m}} B_{m} \\
* & * & * & -\left(\varepsilon_{2} \sum_{i=1}^{m} U_{i}\right)^{-1} & 0 & \cdots & 0 \\
* & * & * & * & -\varepsilon_{2} I & \cdots & 0 \\
& \cdots & & & \cdots & \ddots & \\
* & * & * & * & * & \cdots & -\varepsilon_{2} I
\end{array}\right) \leq 0,
$$

and set $V(t)=x^{T}(t) P x(t)$, where $P=X^{-1}$. Then

$$
V\left(t_{k}\right) \leq \mu V\left(t_{k}^{-}\right) .
$$

Proof. By the second equation of (1), we have

$$
\begin{gathered}
x\left(t_{k}\right)=\left(C\left(t_{k}\right)+\sum_{i=1}^{m} \operatorname{sat}\left(u_{i}\left(t_{k}\right)\right) B_{i}\left(t_{k}\right)\right) x\left(t_{k}^{-}\right) \\
V\left(t_{k}\right)-\mu V\left(t_{k}^{-}\right)=x^{T}\left(t_{k}^{-}\right) \Xi_{1} x\left(t_{k}^{-}\right),
\end{gathered}
$$

where $\Xi_{1}=\left(C(t)+\sum_{i=1}^{m} \operatorname{sat}\left(u_{i}(t)\right) B_{i}(t)\right)^{T} P(C(t)+$ $\left.\sum_{i=1}^{m} \operatorname{sat}\left(u_{i}(t)\right) B_{i}(t)\right)$. By Lemmas 1 and 2, (2), (4), and (8) imply that $\Xi_{1} \leq 0$, hence (9) holds.

Theorem 6. Assume that there exist matrices $X>0, Y$ and positive scalars $\alpha, d, \varepsilon_{1}, \varepsilon_{2}, \varepsilon_{3}$ and $0<\mu<1$ such that (8) and

$$
\left(\begin{array}{cccccc}
\Psi_{1} & A_{1} X & B & Y^{T} & 2 Y^{T} N_{B 1}^{T}+X N_{A}^{T} & D_{1} \\
* & -\alpha X & 0 & 0 & X N_{A 1}^{T} & 0 \\
* & * & -d^{-1} I & 0 & N_{B}^{T} & 0 \\
* & * & * & -d I & 0 & 0 \\
* & * & * & * & -\varepsilon_{3} I & 0 \\
* & * & * & * & * & -\varepsilon_{3}^{-1} I
\end{array}\right)<0
$$

hold, then for any bounded time-delay $\tau(t) \leq \tau$, system (1) can be robustly stabilized by the control law (5) with $K=Y X^{-1}$ for any impulsive time sequence $\left\{t_{k}\right\}$, where $\Psi_{1}=\alpha \mu^{-1} X+A X+$ $X A^{T}+2 B Y+2 Y^{T} B^{T}$.
Proof. Let $P=X^{-1}, \lambda_{0}=\lambda_{\min }(P), \lambda_{1}=\lambda_{\max }(P)$. Substituting $Y=K X^{-1}$ in (11) and pre- and postmultiplying it by $\operatorname{diag}\{P, P, I, I, I, I\}$ yield

$$
\left(\begin{array}{cccccc}
\Psi_{1}^{\prime} & P A_{1} & P B & K^{T} & \left(N_{A}+2 N_{B} K\right)^{T} & P D_{1} \\
* & -\alpha P & 0 & 0 & N_{A 1}^{T} & 0 \\
* & * & -d^{-1} I & 0 & N_{B}^{T} & 0 \\
* & * & * & -d I & 0 & 0 \\
* & * & * & * & -\varepsilon_{3} I & 0 \\
* & * & * & * & * & -\varepsilon_{3}^{-1} I
\end{array}\right)<0,
$$

where $\Psi_{1}^{\prime}=\alpha \mu^{-1} P+(A+2 B K)^{T} P+P(A+2 B K)$. Hence, there exists small positive scalar $\tilde{\mu}$, such that

$$
\left(\begin{array}{cccccc}
\Psi_{1}^{\prime \prime} & P A_{1} & P B & K^{T} & \left(N_{A}+2 N_{B} K\right)^{T} & P D_{1} \\
* & -\alpha \exp (-\tilde{\mu} \tau) P & 0 & 0 & N_{A 1}^{T} & 0 \\
* & * & -d^{-1} I & 0 & N_{B}^{T} & 0 \\
* & * & * & -d I & 0 & 0 \\
* & * & * & * & -\varepsilon_{3} I & 0 \\
* & * & * & * & * & -\varepsilon_{3}^{-1} I
\end{array}\right)<0 \text {, }
$$

where $\Psi_{1}^{\prime \prime}=\left(\alpha \mu^{-1}+\widetilde{\mu}\right) P+(A+2 B K)^{T} P+P(A+2 B K)$.

For given $\rho>0$, choose $0<\delta \leq \sqrt{\mu \lambda_{0} / \lambda_{1}} \rho$. We assume that the initial function $\phi \in P C\left([-\tau, 0], \mathbb{R}^{n}\right)$ satisfying $\|\phi\|_{\tau} \leq \delta$. Set $W(t)=\exp (\tilde{\mu} t) V(t)$. In the following, we will prove that for $t \geq t_{0}-\tau$,

$$
W(t)<\lambda_{0} \rho^{2} .
$$

First, we prove that (14) holds for $t \in\left[t_{0}-\tau, t_{1}\right)$. It is noticed that for $\theta \in[-\tau, 0], W\left(t_{0}+\theta\right) \leq \lambda_{1}\|\phi\|_{\tau}^{2} \leq \mu \lambda_{0} \rho^{2}<$ $\lambda_{0} \rho^{2}$. So it needs only to prove that, for $t \in\left(t_{0}, t_{1}\right),(14)$ holds. Otherwise, there exists $t \in\left(t_{0}, t_{1}\right)$, such that $W(t) \geq \lambda_{0} \rho^{2}$. Set $t^{*}=\inf \left\{t \in\left[t_{0}, t_{1}\right): W(t) \geq \lambda_{0} \rho^{2}\right\}$. Then $t^{*} \in\left(t_{0}, t_{1}\right)$, $W\left(t^{*}\right)=\lambda_{0} \rho^{2}$. Set $\bar{t}=\sup \left\{t \in\left[t_{0}, t^{*}\right): W(t) \leq \mu \lambda_{0} \rho^{2}\right\}$. Then $\bar{t} \in\left[t_{0}, t^{*}\right)$ and $W(\bar{t})=\mu \lambda_{0} \rho^{2}$. Hence, for $t \in\left[\bar{t}, t^{*}\right]$ and $\theta \in[-\tau, 0]$, we have

$$
\mu W(t+\theta) \leq \mu \lambda_{0} \rho^{2} \leq W(t) \leq \lambda_{0} \rho^{2} .
$$


It follows with (6) that

$$
\begin{aligned}
& D^{+} W(t) \\
& \leq \exp (\tilde{\mu} t)\left(\tilde{\mu} V(t)+D^{+} V(t)\right)+\alpha\left(\mu^{-1} W(t)-W(t-\tau(t))\right) \\
& \quad+d^{-1}\left(x^{T}(t) K^{T} K x(t)-\Delta u(t)^{T} \Delta u(t)\right) \exp (\tilde{\mu} t) \\
& \leq \exp (\tilde{\mu} t) \eta^{T}(t) \Xi_{2} \eta(t),
\end{aligned}
$$

where

$$
\begin{aligned}
\eta^{T}(t)= & \left(x^{T}(t), x(t-\tau(t))^{T}, \Delta u(t)^{T}\right) \\
\Xi_{2}= & \left(\begin{array}{ccc}
\Psi_{2}^{\prime} & P A_{1} & P B \\
* & -\alpha \exp (-\tilde{\mu} \tau) P & 0 \\
* & * & -d^{-1} I
\end{array}\right) \\
& +d^{-1}\left(\begin{array}{c}
K^{T} \\
0 \\
0
\end{array}\right)\left(\begin{array}{lll}
K & 0 & 0
\end{array}\right) \\
& +\varepsilon_{3}^{-1}\left(\begin{array}{c}
\left(N_{A}+2 N_{B} K\right)^{T} \\
N_{A 1}^{T} \\
N_{B}^{T}
\end{array}\right)\left(N_{A}+2 N_{B} K \quad N_{A 1} \quad N_{B}\right) \\
& +\varepsilon_{3}\left(\begin{array}{c}
P D_{1} \\
0 \\
0
\end{array}\right)\left(\begin{array}{lll}
D_{1}^{T} P & 0 & 0
\end{array}\right)
\end{aligned}
$$

By Lemma 2, (13) implies that $\Xi_{2}<0$, which yields by (16) that for $t \in\left[\bar{t}, t^{*}\right]$,

$$
D^{+} W(t)<0 \text {. }
$$

It follows that $W\left(t^{*}\right) \leq W(\bar{t})$. This is a contradiction with the fact $W\left(t^{*}\right)=\lambda_{0} \rho^{2}>W(\bar{t})=\mu \lambda_{0} \rho^{2}$. Thus, (14) holds for $t \in\left[t_{0}-\tau, t_{1}\right)$.

Now we assume that (14) holds for $t \in\left[t_{0}-\tau, t_{m}\right)$; we will prove that (14) still holds for $t \in\left[t_{m}, t_{m+1}\right)$. On the contrary, there exists some $t \in\left[t_{m}, t_{m+1}\right)$, such that $W(t)>\lambda_{0} \rho^{2}$. Set $t^{*}=\inf \left\{t \in\left[t_{m}, t_{m+1}\right): W(t) \geq \lambda_{0} \rho^{2}\right\}$. Then because of (9), we have $W\left(t_{m}\right) \leq \mu \lambda_{0} \rho^{2}<\lambda_{0} \rho^{2}$, which implies that $t^{*} \in\left(t_{m}, t_{m+1}\right)$ and $W\left(t^{*}\right)=\lambda_{0} \rho^{2}$. Set $\bar{t}=\sup \left\{t \in\left[t_{m}, t_{*}\right)\right.$ : $\left.W(t) \leq \mu \lambda_{0} \rho^{2}\right\}$. Then $\bar{t} \in\left[t_{m}, t^{*}\right)$ and $W(\bar{t})=\mu \lambda_{0} \rho^{2}$. Hence, for $t \in\left[\bar{t}, t^{*}\right]$, we obtain that (15) holds, which leads that (16) and (18) hold. It follows that $W\left(t^{*}\right) \leq W(\bar{t})$, which contradicts the fact $W\left(t^{*}\right)=\lambda_{0} \rho^{2}>W(\bar{t})=\mu \lambda_{0} \rho^{2}$. Thus, (14) holds for $t \in\left[t_{0}-\tau, t_{m+1}\right)$. By mathematical induction, (14) holds for $t \geq t_{0}-\tau$. That is, system (1) can be robustly stabilized.

Remark 7. By Theorem 6, in the case $\mu<1$, different from Chen and Zheng [7], we can stabilize impulsive systems with any time sequences by saturation control.
Remark 8. In Chen and Zheng [7], the authors need continuous control input $u_{c}(t)$ and impulsive control input $u_{d}(t)$. But in this paper, we only need continuous control input $u(t)$.

For $\mu=1$, we have the following result.

Theorem 9. Assume that there exist matrices $X>0, Y$ and positive scalars $\alpha, d, \varepsilon_{1}, \varepsilon_{2}$, and $\varepsilon_{3}$ such that (8) and (11) hold, then for any bounded time-delay $\tau(t) \leq \tau$, system (1) can be robustly stabilized by the control law (5) with $K=Y X^{-1}$ for any impulsive time sequence $\left\{t_{k}\right\}$, where $\Psi_{2}=\alpha X+A X+$ $X A^{T}+2 B Y+2 Y^{T} B^{T}$.

Proof. Choose a sufficiently small scalar $h>0$, and set $\bar{\mu}=1-h$. Then for $\|\phi\|_{\tau} \leq \delta \leq \sqrt{\bar{\mu} \lambda_{0} / \lambda_{1}} \rho$ with $\phi \in$ $P C\left([-\tau, 0], \mathbb{R}^{n}\right)$, similar to the proof of Theorem 6 , we can obtain the result of Theorem 9. The proof is complete.

For $\mu>1$, we have the following result.

Theorem 10. Assume that for a prescribed scalar $\beta>0$, there exist matrices $X>0, Y$ and positive scalars $\alpha, d, \varepsilon_{1}, \varepsilon_{2}, \varepsilon_{3}$, and $\mu>1$ such that (8) and

$$
\left(\begin{array}{cccccc}
\Psi_{3} & A_{1} X & B & Y^{T} & 2 Y^{T} N_{B}^{T}+X N_{A}^{T} & D_{1} \\
* & -\alpha X & 0 & 0 & X N_{A 1}^{T} & 0 \\
* & * & -d^{-1} I & 0 & N_{B}^{T} & 0 \\
* & * & * & -d I & 0 & 0 \\
* & * & * & * & -\varepsilon_{3} I & 0 \\
* & * & * & * & * & -\varepsilon_{3}^{-1} I
\end{array}\right)<0
$$

hold, then for any bounded time-delay $\tau(t) \leq \tau$, there exists a positive scalar $h$, such that system (1) can be robustly stabilized by the control law (5) with $K=Y X^{-1}$ over time sequence $\mathcal{N}_{\text {min }}(\beta-h)$, where $\Psi_{3}=\left(\beta^{-1} \ln \mu+\alpha \mu\right) X+A X+X A^{T}+$ $2 B Y+2 Y^{T} B^{T}$.

Proof. Let $P=X^{-1}, \lambda_{0}=\lambda_{\min }(P), \lambda_{1}=\lambda_{\max }(P)$, and $V(t)=x^{T}(t) P x(t)$. Substituting $Y=K X^{-1}$ in (19) and preand postmultiplying it by $\operatorname{diag}\{P, P, I, I, I, I\}$ yield

$$
\left(\begin{array}{cccccc}
\Psi_{3}^{\prime} & P A_{1} & P B & K^{T} & \left(N_{A}+2 N_{B} K\right)^{T} & P D_{1} \\
* & -\alpha P & 0 & 0 & N_{A 1}^{T} & 0 \\
* & * & -d^{-1} I & 0 & N_{B}^{T} & 0 \\
* & * & * & -d I & 0 & 0 \\
* & * & * & * & -\varepsilon_{3} I & 0 \\
* & * & * & * & * & -\varepsilon_{3}^{-1} I
\end{array}\right)<0
$$


where $\Psi_{3}^{\prime}=\left(\beta^{-1} \ln \mu+\alpha \mu\right) P+(A+2 B K)^{T} P+P(A+2 B K)$. Hence, there exist small positive scalars $\tilde{\mu}$ and $h$, such that

$$
\left(\begin{array}{cccccc}
\Psi_{3}^{\prime \prime} & P A_{1} & P B & K^{T} & \left(N_{A}+2 N_{B} K\right)^{T} & P D_{1} \\
* & -\alpha \exp (-\tilde{\mu} \tau) P & 0 & 0 & N_{A 1}^{T} & 0 \\
* & * & -d^{-1} I & 0 & N_{B}^{T} & 0 \\
* & * & * & -d I & 0 & 0 \\
* & * & * & * & -\varepsilon_{3} I & 0 \\
* & * & * & * & * & -\varepsilon_{3}^{-1} I
\end{array}\right)<0
$$

where $\Psi_{3}^{\prime \prime}=\left(\mu_{1}+\alpha \mu+\tilde{\mu}\right) P+(A+2 B K)^{T} P+P(A+2 B K)$, $\mu_{1}=(\beta-h)^{-1} \ln \mu$.

For given $\rho>0$, choose $0<\delta \leq \sqrt{\lambda_{0} / \mu \lambda_{1}} \rho$. We assume that the initial function $\phi \in P C\left([-\tau, 0], \mathbb{R}^{n}\right)$ satisfying $\|\phi\|_{\tau} \leq \delta$. Set $W(t)=\exp (\tilde{\mu} t) V(t)$. In the following, we will prove that for $t \geq t_{0}-\tau,(14)$ holds. First, we prove that (14) holds for $t \in\left[t_{0}-\tau, t_{1}\right)$. It is noticed that for $\theta \in[-\tau, 0]$, $W\left(t_{0}+\theta\right) \leq \lambda_{1}\|\phi\|_{\tau}^{2} \leq \mu^{-1} \lambda_{0} \rho^{2}<\lambda_{0} \rho^{2}$. Similar to the proof of Theorem 6 , it needs only to prove that, for $t \in\left(t_{0}, t_{1}\right)$, (14) holds. Otherwise, there exists $t \in\left(t_{0}, t_{1}\right)$, such that $W(t) \geq \lambda_{0} \rho^{2}$. Set $t^{*}=\inf \left\{t \in\left[t_{0}, t_{1}\right): W(t) \geq \lambda_{0} \rho^{2}\right\}$. Then $t^{*} \in\left(t_{0}, t_{1}\right), W\left(t^{*}\right)=\lambda_{0} \rho^{2}$. Set $\bar{t}=\sup \left\{t \in\left[t_{0}, t^{*}\right): W(t) \leq\right.$ $\left.\mu^{-1} \lambda_{0} \rho^{2}\right\}$. Then $\bar{t} \in\left[t_{0}, t^{*}\right)$ and $W(\bar{t})=\mu^{-1} \lambda_{0} \rho^{2}$. Hence, for $t \in\left[\bar{t}, t^{*}\right]$ and $\theta \in[-\tau, 0]$, we have

$$
W(t+\theta) \leq \lambda_{0} \rho^{2} \leq \mu W(t) .
$$

By (6), (7), and (22), we have

$$
\begin{aligned}
D^{+} & W(t) \\
\leq & \exp (\tilde{\mu} t)\left(\tilde{\mu} V(t)+D^{+} V(t)\right)+\alpha(\mu W(t)-W(t-\tau(t))) \\
& +d^{-1}\left(x^{T}(t) K^{T} K x(t)-\Delta u(t)^{T} \Delta u(t)\right) \exp (\tilde{\mu} t) \\
\leq & \exp (\tilde{\mu} t) \eta^{T}(t) \Xi_{3} \eta(t)-\mu_{1} W(t),
\end{aligned}
$$

where

$$
\begin{aligned}
\Xi_{3}= & \left(\begin{array}{ccc}
\Psi_{3}^{\prime \prime} & P A_{1} & P B \\
* & -\alpha \exp (-\tilde{\mu} \tau) P & 0 \\
* & * & -d^{-1} I
\end{array}\right) \\
& +d^{-1}\left(\begin{array}{c}
K^{T} \\
0 \\
0
\end{array}\right)\left(\begin{array}{lll}
K & 0 & 0
\end{array}\right) \\
& +\varepsilon_{3}^{-1}\left(\begin{array}{c}
\left(N_{A}+2 N_{B} K\right)^{T} \\
N_{A 1}^{T} \\
N_{B}^{T}
\end{array}\right)\left(N_{A}+2 N_{B} K N_{A 1} N_{B}\right) \\
& +\varepsilon_{3}\left(\begin{array}{c}
P D_{1} \\
0 \\
0
\end{array}\right)\left(\begin{array}{lll}
D_{1}^{T} P & 0 & 0
\end{array}\right),
\end{aligned}
$$

By Lemma 2, (21) implies that $\Xi_{3}<0$, which yields by (23) that for $t \in\left[\bar{t}, t^{*}\right]$,

$$
D^{+} W(t)<-\mu_{1} W(t) .
$$

It follows that $W\left(t^{*}\right) \leq W(\bar{t})$. This is a contradiction with the fact $W\left(t^{*}\right)=\lambda_{0} \rho^{2}>W(\bar{t})=\mu^{-1} \lambda_{0} \rho^{2}$. Thus, (14) holds for $t \in\left[t_{0}-\tau, t_{1}\right)$.

Now we assume that (14) holds for $t \in\left[t_{0}-\tau, t_{m}\right)$; we will prove that (14) still holds for $t \in\left[t_{m}, t_{m+1}\right)$. First, we claim that $W\left(t_{m}^{-}\right) \leq \mu^{-1} \lambda_{0} \rho^{2}$. Otherwise, we have $W\left(t_{m}^{-}\right)>$ $\mu^{-1} \lambda_{0} \rho^{2}$. Here we must consider two cases.

Case 1. $W(t)>\mu^{-1} \lambda_{0} \rho^{2}$ for all $t \in\left[t_{m-1}, t_{m}\right)$. It follows that (22) holds according to the fact that (14) holds for $t \in\left[t_{0}-\right.$ $\left.\tau, t_{m}\right)$. Hence (23) and (25) hold for $t \in\left[t_{m-1}, t_{m}\right)$. They lead that

$$
\begin{aligned}
W\left(t_{m}^{-}\right) & \leq W\left(t_{m-1}\right) \exp \left(-\mu_{1}\left(t_{m}-t_{m-1}\right)\right) \\
& \leq \lambda_{0} \rho^{2} \exp \left(-\mu_{1}(\beta-h)\right)=\mu^{-1} \lambda_{0} \rho^{2} .
\end{aligned}
$$

This is also a contradiction.

Case 2. There exists some $t \in\left[t_{m-1}, t_{m}\right)$, such that $W(t) \leq$ $\mu^{-1} \lambda_{0} \rho^{2}$. Set $\bar{t}=\sup \left\{t \in\left[t_{m-1}, t_{m}\right): W(t) \leq \mu^{-1} \lambda_{0} \rho^{2}\right\}$. Then $\bar{t} \in\left[t_{m-1}, t_{m}\right), W(\bar{t})=\mu^{-1} \lambda_{0} \rho^{2}$ and (22) for $t \in\left[\bar{t}, t_{m}\right]$. It follows that (23) and (25) hold, which yields that $W\left(t_{m}^{-}\right) \leq$ $W(\bar{t})=\mu^{-1} \lambda_{0} \rho^{2}$. This is a contradiction which implies that $W\left(t_{m}^{-}\right) \leq \mu^{-1} \lambda_{0} \rho^{2}$, and hence by (9), we obtain that $W\left(t_{m}\right) \leq$ $\mu W\left(t_{m}^{-}\right) \leq \lambda_{0} \rho^{2}$.

Now we assume that (14) is not true for $t \in\left[t_{m}, t_{m+1}\right)$. Set $t^{*}=\inf \left\{t \in\left[t_{m}, t_{m+1}\right): W(t) \geq \lambda_{0} \rho^{2}\right\}$. Then $t^{*} \in\left[t_{m}, t_{m+1}\right)$ and $W\left(t^{*}\right)=\lambda_{0} \rho^{2}$. If there exists some $t \in\left[t_{m}, t^{*}\right)$, such that $W(t) \leq \mu^{-1} \lambda_{0} \rho^{2}$, then set $\bar{t}=\sup \left\{t \in\left[t_{m}, t^{*}\right): W(t) \leq\right.$ $\left.\mu^{-1} \lambda_{0} \rho^{2}\right\}$, otherwise, we set $\bar{t}=t_{m}$. Then $\bar{t} \in\left[t_{m}, t^{*}\right)$. W( $\left.\bar{t}\right)<$ $\lambda_{0} \rho^{2}$. It follows that (22), (23), and (25) hold, which yields that $W\left(t^{*}\right) \leq W(\bar{t})<\lambda_{0} \rho^{2}$. This is a contradiction, which implies that (14) is true for $t \in\left[t_{m}, t_{m+1}\right)$. By mathematical induction, (14) holds for $t \geq t_{0}-\tau$. That is, system (1) can be robustly stabilized over time sequence $\mathcal{N}_{\min }(\beta-h)$.

Remark 11. On the conditions in Theorem 10, with the prescribed scalar $\beta>0$, we can stabilize not only the impulsive systems over time sequence $\mathcal{N}_{\min }(\beta)$, but some ones over other time sequences.

\section{Numerical Examples}

In this section, two illustrative examples will be presented to show the effectiveness of the results obtained.

Example 12. Consider the uncertain impulsive time-delay systems (1) with the following specifications: $\mu=0.8, d=1$, $\alpha=2, \varepsilon_{1}=0.3034, \varepsilon_{2}=0.5, \varepsilon_{3}=0.5, U_{1}=U_{2}=1,0 \leq$ $\tau(t) \leq \gamma=$ : const, $A=\left(\begin{array}{cc}-4 & 0.2 \\ 0.1 & -5\end{array}\right), A_{1}=\left(\begin{array}{cc}1 & 0 \\ 0.2 & 1\end{array}\right), B=\left(\begin{array}{cc}1 & 0.1 \\ 0.2 & 1\end{array}\right)$, $C=\left(\begin{array}{cc}-0.1 & 0 \\ -0.2 & -0.1\end{array}\right), N_{A}=\left(\begin{array}{cc}0.1 & 0 \\ 1 & 0.1\end{array}\right), N_{A 1}=\left(\begin{array}{cc}0.1 & 0 \\ 0.1 & 0.1\end{array}\right), N_{B}=$ $\left(\begin{array}{cc}0.01 & 0 \\ 0.1 & 0.1\end{array}\right), N_{C}=\left(\begin{array}{cc}0.1 & 0.1 \\ 0 & 0.1\end{array}\right), B_{1}=\left(\begin{array}{cc}0.1 & 0 \\ -1 & 0.1\end{array}\right), B_{2}=\left(\begin{array}{cc}0.1 & 0.2 \\ 0 & 0.1\end{array}\right), N_{B 1}=$ $\left(\begin{array}{cc}0.1 & -0.1 \\ 0 & 0.1\end{array}\right), N_{B 2}=\left(\begin{array}{cc}0.1 & 0.1 \\ 0 & 0.1\end{array}\right), D_{1}=\left(\begin{array}{ll}1 & 0.1 \\ 0 & 1\end{array}\right), D_{2}=\left(\begin{array}{ll}1 & 0.1 \\ 0 & 1\end{array}\right)$. Then 
by solving LMIs (8) and (11), we obtain the following results:

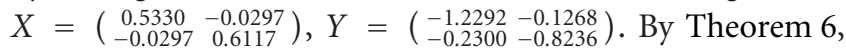
the controller $u(t)=Y X^{-1} x(t)=\left(\begin{array}{l}-2.3241-0.3201 \\ -0.5079\end{array}-1.3710\right) x(t)$ is obtained. It exponentially stabilizes the impulsive system over any impulsive time sequence according to Theorem 6.

Example 13. Consider the uncertain impulsive time-delay systems (1) with the following specifications: $\mu=1, d=1$, $\alpha=2, \varepsilon_{1}=0.4049, \varepsilon_{2}=0.5, \varepsilon_{3}=0.5, U_{1}=U_{2}=1,0 \leq$ $\tau(t) \leq \gamma=$ : const, $A=\left(\begin{array}{cc}-3 & 0.2 \\ 0.1 & -5\end{array}\right), A_{1}=\left(\begin{array}{cc}1 & 0 \\ 0.2 & 1\end{array}\right), B=\left(\begin{array}{cc}1 & 0.2 \\ 0.2 & 0.7\end{array}\right)$, $C=\left(\begin{array}{cc}0.1 & 0 \\ -0.2 & -0.1\end{array}\right), N_{A}=\left(\begin{array}{cc}0.1 & 0.2 \\ 1 & 0.1\end{array}\right), N_{A 1}=\left(\begin{array}{cc}0.1 & 0 \\ 1 & 0.1\end{array}\right), N_{B}=$ $\left(\begin{array}{cc}0.1 & 0 \\ 0.2 & 0.1\end{array}\right), N_{C}=\left(\begin{array}{cc}0.1 & 0.1 \\ 0 & 0.1\end{array}\right), B_{1}=\left(\begin{array}{cc}0.1 & 0 \\ -1 & 0.1\end{array}\right), B_{2}=\left(\begin{array}{cc}0.1 & 0.2 \\ 0 & 0.1\end{array}\right), N_{B 1}=$ $\left(\begin{array}{cc}0.1 & -0.1 \\ 0 & 0.1\end{array}\right), N_{B 2}=\left(\begin{array}{cc}0.1 & 0.1 \\ 0 & 0.1\end{array}\right), D_{1}=\left(\begin{array}{cc}1 & 0.1 \\ 1 & 1\end{array}\right), D_{2}=\left(\begin{array}{cc}1 & 0.1 \\ 0.2 & 1\end{array}\right)$. Then by solving LMIs (8) and (11), we obtain the following results: $X=\left(\begin{array}{cc}0.7144 & -0.0180 \\ -0.0180 & 0.8380\end{array}\right), Y=\left(\begin{array}{cc}-2.4545 & -0.8386 \\ -0.6829 & -1.5880\end{array}\right)$. By Theorem 6, the controller $u(t)=Y X^{-1} x(t)=\left(\begin{array}{l}-3.4628-1.0751 \\ -1.0042\end{array}-1.9166\right) x(t)$ is obtained. It exponentially stabilizes the impulsive system over any impulsive time sequence according to Theorem 6.

Example 14. Consider the uncertain impulsive time-delay systems (1) with the following specifications: $\mu=1.5$, $d=1, \alpha=2, \varepsilon_{1}=0.1100, \varepsilon_{2}=0.5, \varepsilon_{3}=0.5, U_{1}=$ $U_{2}=1,0 \leq \tau(t) \leq \gamma=$ : const, $A=-10 I, A_{1}=$ $B=D_{1}=I, C=-0.1 I, N_{A}=N_{A 1}=N_{C}=0.1 I$, $N_{B}=B_{1}=N_{B 1}=N_{B 2}=0.1 I, B_{2}=\operatorname{diag}\{1,0.1\}$, $D_{2}=\operatorname{diag}\{3,1\}$. Then by solving LMIs (8) and (19), we obtain that $\beta=0.4055, X=\operatorname{diag}\{1.2150,1.0491\}, Y=$ $\operatorname{diag}\{0.7386,0.4180\}$. By Theorem 10, the controller $u(t)=$ $Y X^{-1} x(t)=\left(\begin{array}{cc}0.6079 & 0 \\ 0 & 0.3984\end{array}\right) x(t)$ is obtained. It exponentially stabilizes the impulsive system over impulsive time sequence $\mathcal{N}_{\text {min }}(0.4055-h)$ according to Theorem 10 , where $h>0$ is some small scalar. In fact, we can obtain some $h \geq 0.002$.

\section{Conclusion}

This paper studied a class of uncertain impulsive time-delay systems. Based on Lyapunov function and Razumikhintype techniques, we obtain conditions which can guarantee the above systems robustly exponentially stabiliable for any impulsive time sequences and improves some related results.

\section{Acknowledgments}

The authors would like to thank the editor and the reviewers for their valuable comments and suggestions which helped to significantly improve the paper. This work is supported by Distinguished Expert Science Foundation of Naval Aeronautical and Astronautical University.

\section{References}

[1] V. Lakshmikantham, D. D. Bainov, and P. S. Simeonov, Theoery of Impulsive Differential Equations, World Scientific, Singapore, 1989.

[2] D. D. Bainov and P. S. Simeonov, Systems with Impulsive Effect: Stability, Theory and Applications, Ellis Horwood, Chichester, UK, 1989.
[3] Z. Li, C. B. Soh, and X. Xu, "Stability of impulsive differential systems," Journal of Mathematical Analysis and Applications, vol. 216, no. 2, pp. 644-653, 1997.

[4] Z. Li, C. B. Soh, and X. Xu, "Robust stability of a class of hybrid dynamic uncertain systems," International Journal of Robust and Nonlinear Control, vol. 8, no. 12, pp. 1059-1072, 1998.

[5] Z. G. Li, C. Y. Wen, and Y. C. Soh, "Analysis and design of impulsive control systems," IEEE Transactions on Automatic Control, vol. 46, no. 6, pp. 894-897, 2001.

[6] T. Yang, Impulsive Systems and Control: Theory and Applications, Nova Science, New York, NY, USA, 2001.

[7] W. H. Chen and W. X. Zheng, "Robust stability and $H_{\infty}$ control of uncertain impulsive systems with time-delay," Automatica, vol. 45, no. 1, pp. 109-117, 2009.

[8] A. Barreiro and A. Baños, "Delay-dependent stability of reset systems," Automatica, vol. 46, no. 1, pp. 216-221, 2010.

[9] H. Xu and K. L. Teo, "Robust stabilization of uncertain impulsive switched systems with delayed control," Computers and Mathematics with Applications, vol. 56, no. 1, pp. 63-70, 2008.

[10] Y. Liu, A. Eberhard, and K. L. Teo, "A Numerical method for a class of mixed switching and impulsive optimal control problems," Computers and Mathematics with Applications, vol. 52, no. 5, pp. 625-636, 2006.

[11] S. Ma and C. Zhang, "Robust stability and $H_{\infty}$ control for uncertain discrete Markovian jump singular systems with mode-dependent time-delay," International Journal of Robust and Nonlinear Control, vol. 19, no. 9, pp. 965-985, 2009.

[12] A. T. Fuller, "In-the-large stability of relay and saturating control systems with linear controllers," International Journal of Control, vol. 10, no. 4, pp. 457-480, 1969.

[13] E. D. Sontag and H. J. Sussmann, "Nonlinear output feedback design for linear systems with saturating controls," in Proceedings of the 29th IEEE Conference on Decision and Control, pp. 3414-3416, December 1990.

[14] H. Y. Su, J. Chu, R. Q. Lu, and X. F. Ji, Robust Control Theory of Uncertain Systems with Timedelays, Science Press, Beijing, China, 2007. 

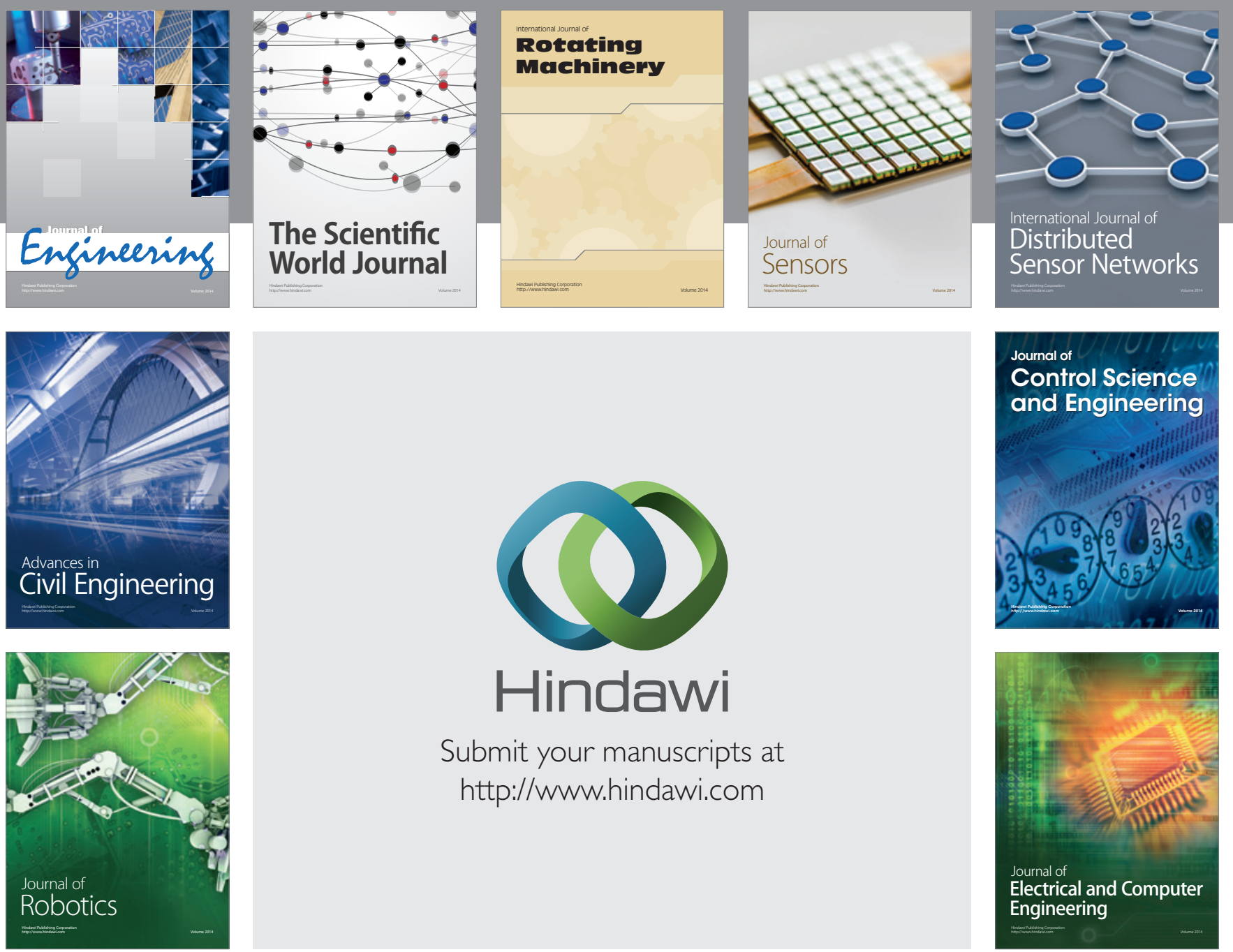

Submit your manuscripts at

http://www.hindawi.com
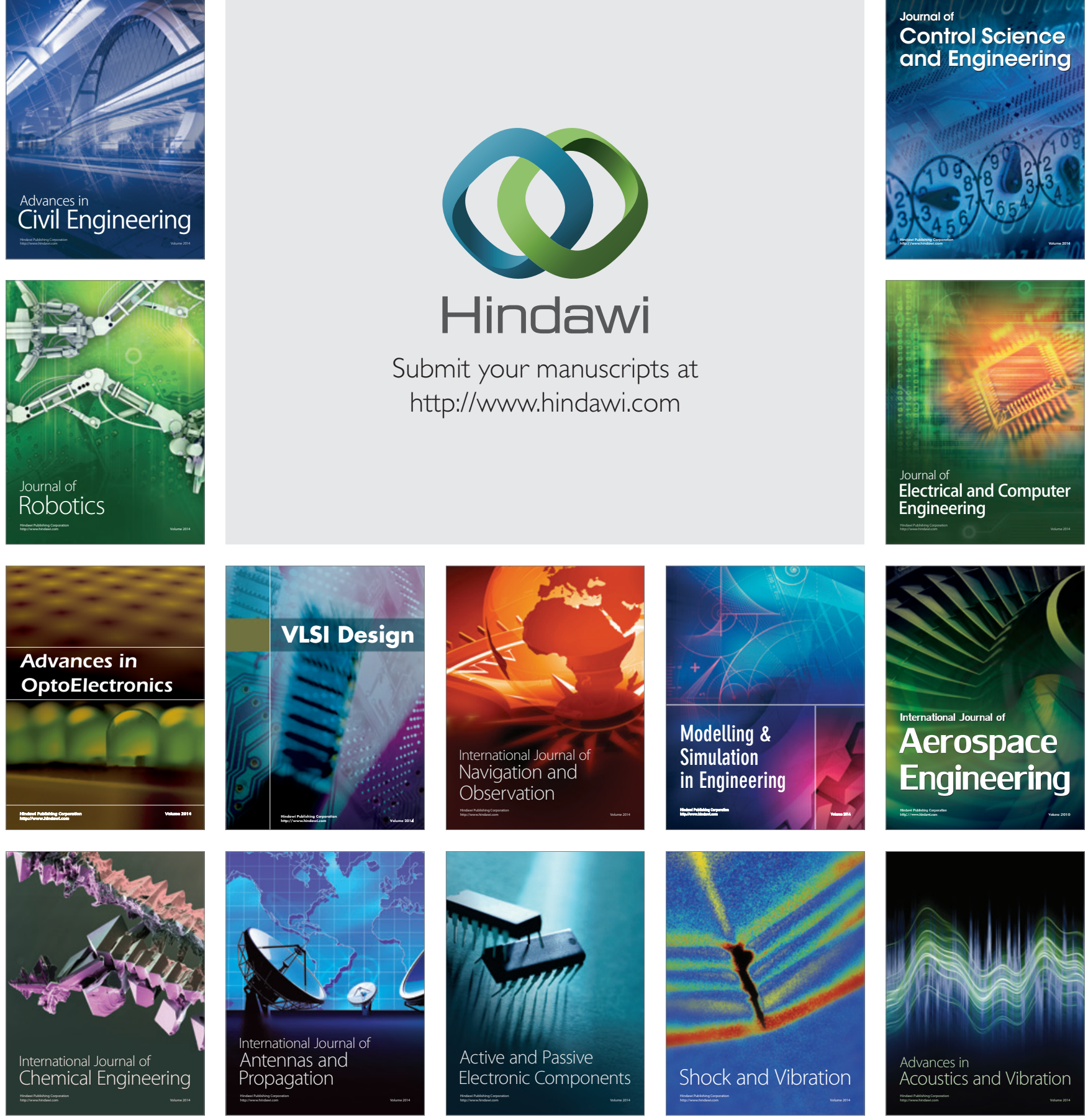